\title{
Time to Extubation-What Causes the Variations?
}

\author{
Preet Mohinder Singh ${ }^{1}$ (D) Anuradha Borle ${ }^{1} \cdot$ Jason McGavin $^{2} \cdot$ Anjan Trikha $^{1} \cdot$ \\ Ashish Sinha ${ }^{2}$
}

Published online: 28 October 2017

(C) Springer Science+Business Media, LLC 2017

\section{Time to Extubation-What Causes the Variations?}

We thank Dexter et al. for their interest in our paper. The primary goal of our meta-analysis was to evaluate the differences in the recovery profile of patients undergoing bariatric surgery when sevoflurane or desflurane is used for maintenance of general anesthesia. We presented our findings of high heterogeneity that occurred in the pooled results and did not mathematically explore the causes for it [1]. Unlike the manuscript cited by the authors, we did not use any previous data to construct a distribution of "time to extubation" [2] and thus are in no position to comment upon suggested possibilities. Although intuitively we agree to their comment and this can certainly form one of the objectives to be explored for future research on this topic.

Preet Mohinder Singh

Preetrajpal@gmail.com

Anuradha Borle

Andromeda85@gmail.com

Jason McGavin

jmcgavin12@gmail.com

Anjan Trikha

anjantrikha@gmail.com

Ashish Sinha

Ashish.Sinha@tuhs.temple.edu

1 Department of Anesthesia, All India Institute of Medical Sciences, New Delhi 110029, India

2 Anesthesiology and Perioperative, Medicine, Lewis Katz School of Medicine, Temple, University Health System,

Philadelphia, PA 19140, USA
We agree to the fact that for economic analysis based upon high heterogeneity, a proportional reduction of time plays more significant role than actual absolute value of decrease in time to extubation variable. Further, it would not be fully appropriate to completely rule out methodological variations across the trials as a contributor towards the high heterogeneity. The included trials in our meta-analysis had variations in the type of bariatric procedures itself. A sleeve gastrectomy is likely to be a much shorter procedure than a diversion procedure. Thus, marked variations in total procedure durations did certainly exist across the trials we included. Dexter et al. in their letter also state that one of the predictors of time to extubation is the total duration of procedure itself and such variations did exist in our included trials. We however agree to the possibility proposed by Dexter et al. in their letter that analysis of time to tracheal extubation can become more informative if one can incorporate the probability distribution.

\section{Compliance with Ethical Standards}

Conflict of Interest The authors declare that they have no conflict of interest.

\section{References}

1. Singh PM, Borle A, McGavin J, et al. Comparison of the recovery profile between desflurane and sevoflurane in patients undergoing bariatric surgery - a meta-analysis of randomized controlled trials. Obes Surg. 2017;27(11):3031-9.

2. Dexter F, Bayman EO, Epstein RH. Statistical modeling of average and variability of time to extubation for meta-analysis comparing desflurane to sevoflurane. Anesth Analg. 2010;110(2): 570-80. 